\title{
PHASE RETRIEVAL BY USING TRANSPORT-OF-INTENSITY EQUATION AND DIFFERENTIAL INTERFERENCE CONTRAST MICROSCOPY
}

\author{
Emrah Bostan $^{\S \star}$, Emmanuel Froustey ${ }^{\S \star}$, Benjamin Rappaz $z^{\dagger}$ Etienne Shaffer ${ }^{\ddagger}$, Daniel Sage ${ }^{\S}$, and Michael Unser ${ }^{\S}$ \\ ${ }^{\S}$ Biomedical Imaging Group, EPFL, Lausanne, Switzerland \\ ${ }^{\dagger}$ Biomolecular Screening Facility, EPFL, Lausanne, Switzerland \\ ${ }^{\ddagger}$ Microsystems Laboratory 1, EPFL, Lausanne, Switzerland
}

\begin{abstract}
We present a variational reconstruction algorithm for the phaseretrieval problem by using the differential interference contrast microscopy. Principally, we rely on the transport-of-intensity equation that specifies the sought phase as the solution of a partial differential equation. Our approach is based on an iterative reconstruction algorithm involving the total variation regularisation which is efficiently solved via the alternating direction method of multipliers. We illustrate the applicability of the method via real data experiments. To the best of our knowledge, this work demonstrates the performance of such an iterative algorithm on real data for the first time.
\end{abstract}

Index Terms-Phase retrieval, transport-of-intensity equation,sparse reconstruction, total variation regularisation.

\section{INTRODUCTION}

Reconstructing the phase of a complex field given its intensity is a fundamental problem in bioimaging. Most cells and soft tissues are highly transparent. Thus, without staining or tagging, they generate very low contrast intensity images. This implies that they are barely visible under a standard brightlight microscope. Knowing that such specimens change the phase of the light wave (i.e. shape and structure of the specimen is encoded in the phase image) has led to the development of many well-established imaging modalities [1].

Differing from the instrumentation-based solutions, the transport-of-intensity equation (TIE) serves as a purely computational approach [2]. Originating from the parabolic wave equation, TIE links the phase image to the variations in the intensity induced by wave propagation. The essence of this method is to measure the intensity along the propagation direction at multiple (three in our case) positions. In mathematical terms, the relationship between these measurements and the phase image is expressed via an inhomogeneous second-order differential equation whose solution is unique (up to an additive constant) [3].

This work was supported by the European Research Council under Grant ERC-2010-AdG 267439-FUN-SP

* These authors contributed equally to this work.
An advantage of TIE is that the resulting phase does not need to be unwrapped, as is common for many other interferometric methods [4]. Allowing phase images to be obtained by using numerical methods-rather than implementing specialised hardware modifications-TIE-based imaging is a viable tool for electron microscopy [3] and X-ray imaging [5]. In addition, TIE can be applied to a partially coherent source, even though it has been initially derived for coherent illumination [6]. This makes it applicable to a practical differential interference contrast (DIC) microscope [7].

The common practice for resolving TIE has been the direct inversion of the model (with appropriate boundary conditions) $[3,5,7]$. Although this approach is non-iterative, it is extremely sensitive to the measurement noise since the said inversion is characterised by an integral operator. Consequently, the reconstructed phase images suffer from noise amplification. This observation strongly motivates the development of reconstruction algorithms that are resilient to noise. Also, the reconstruction performance can be further improved by imposing suitable characteristics on the solution.

The main contributions of the present work are as follows:

- The variational formulation of the phase reconstruction problem by utilising TIE for DIC microscopy. The proposed method incorporates total variation (TV) regularisation which allows for preservation of abrupt phase transitions.

- An iterative reconstruction algorithm, based on the alternating direction method of multipliers (ADMM), that decomposes the optimisation into sub-problems that are solved efficiently.

- The validation of the method by applying it to experimentally-acquired DIC data.

The remainder of the paper is organised as follows: In Section 2, we start by explaining the underlying mathematical scheme that constitutes our forward model. In Section 3, we state an inverse problem formulation and explain our iterative algorithm. We conduct experiments where we reconstruct phase images from a series of DIC images of HeLa cells in Section 4. 


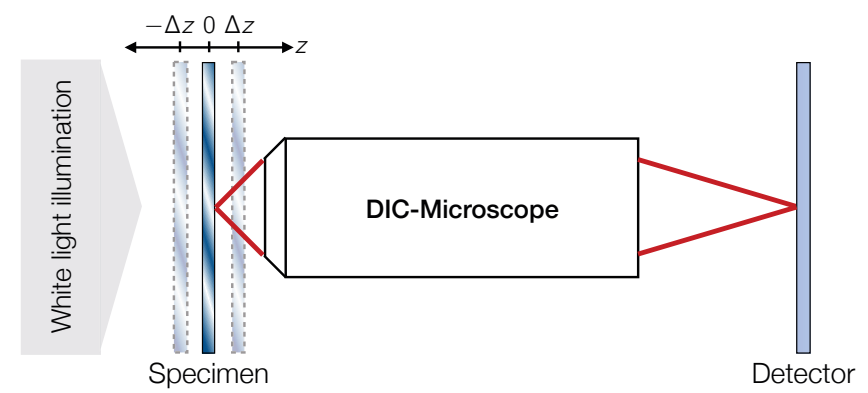

Fig. 1. Simplified representation of our approach for optical phase retrieval via transport-of-intensity equation.

\section{PHYSICAL MODEL}

\subsection{Transport-of-Intensity Equation}

Consider a monochromatic, scalar field at some axial plane $z \in \mathbb{R}$, over some closed and bounded transverse domain. This field is propagating in the $+z$ direction and is represented by

$$
U(\mathbf{x}, z)=U_{0}(\mathbf{x}, z) \mathrm{e}^{\mathrm{j} k z},
$$

where $U_{0}$ is the complex-amplitude with $\mathbf{x}=\left(x_{1}, x_{2}\right) \in$ $\mathbb{R}^{2}$, and $k=2 \pi / \lambda$ is the wave number with $\lambda$ being the wavelength.

Under the paraxial approximation, which is to say that the propagation is dominantly along the $z$-axis and the dispersion in the transverse plane is negligible, we have

$$
U_{0}(\mathbf{x}, z)=\sqrt{I(\mathbf{x}, z)} \mathrm{e}^{\mathrm{j} \phi(\mathbf{x}, z)},
$$

where $I$ and $\phi$ denote the intensity and the phase, respectively. Note that any physical measurement yields $\left|U_{0}\right|^{2}=I$, so the phase information is lost.

To recover the phase, Teague [2] has derived the so-called transport-of-intensity equation (TIE):

$$
-k \frac{\partial}{\partial z} I(\mathbf{x}, z)=\nabla_{\perp} \cdot I(\mathbf{x}, z) \nabla_{\perp} \phi(\mathbf{x}, z),
$$

where $\nabla_{\perp}$ is the transverse gradient operator and · denotes the dot product. The remarkable aspect of (2) is that it establishes a direct mathematical link between two physical quantities, namely the spatial phase and the axial derivative of the intensity of the field.

For bioimaging applications, one usually assumes that $U_{0}$ represents the field leaving a thin, phase-only object - this is reasonable for most biological samples such as cells and microorganisms - that is illuminated uniformly. This means that $I(\mathbf{x}, z)$ is constant over $\mathbf{x}$ so that (2) is rewritten as

$$
-\frac{k}{I(\mathbf{x}, z)} \frac{\partial}{\partial z} I(\mathbf{x}, z)=\nabla_{\perp}^{2} \phi(\mathbf{x}, z)
$$

where $\nabla_{\perp}^{2}$ denotes the two-dimensional Laplacian over the transverse plane.
Practically speaking, the axial derivative of the intensity is approximated by finite differences. One possible approximation is carried out by using two equispaced defocused measurements:

$$
\frac{\partial}{\partial z} I(\mathbf{x}, z) \approx \frac{I(\mathbf{x}, z+\Delta z)-I(\mathbf{x}, z-\Delta z)}{2 \Delta z},
$$

where $\Delta z$ is the defocus amount. That being the case, (3) indicates experimental access to the Laplacian of the phase by acquiring three images.

In microscopic imaging applications, the defocused measurements are recorded via moving the stage of the microscope (see Figure 1), which is equivalent to displacing the detector along the optical axis. Therefore, by solving (3), one retrieves the phase at the detector plane which, assuming a perfect imaging system, corresponds to a magnified version of $U_{0}$.

\subsection{Differential Interference Contrast Microscopy}

We are now interested in combining the TIE formalism with differential interference contrast (DIC) images. Fundamentally, DIC microscope relies on polarising optics to produce two orthogonally polarised beams. These beams traverse the specimen small distance apart from each other. Hence, they perceive the same phase profile with a very small displacement. Then, the beams are optically interfered such that the intensity image — related to the derivative of the phase - has increased contrast [8].

Let us denote the differently polarised fields by $U_{1}$ and $U_{2}$ and their intensities by $I_{1}$ and $I_{2}$. We first note that (2) is satisfied for both $U_{1}$ and $U_{2}$. Again under uniform illumination and thin sample assumptions, it further holds that $I_{1}=I_{2}=I$. Considering these along with the image formation in DIC, Kou et al. [7] have developed the following model:

$$
\frac{-k \mathrm{C}}{I_{\mathrm{DIC}}(\mathbf{x}, z)} \frac{\partial}{\partial z} I_{\mathrm{DIC}}(\mathbf{x}, z)=\nabla_{\perp}^{2} \phi(\mathbf{x}, z),
$$

where $I_{\mathrm{DIC}}$ is the intensity image recorded by the DIC microscope. In (5), C is a constant depending on the bias value introduced by the microscope and is known to the practitioner. By looking at (5), we see that the TIE technique can be readily extended to DIC imaging.

For solving (5), Kou et al. [7] have applied the inverse operator

$$
\left(\nabla_{\perp}^{2}\right)^{-1} \stackrel{\mathcal{F}}{\longleftrightarrow}\|\boldsymbol{\omega}\|^{-2}
$$

with $\boldsymbol{\omega}=\left(\omega_{1}, \omega_{2}\right)$ representing the spatial frequency. The singularity at the origin is handled with a constant treatment: $\|\boldsymbol{\omega}\|^{-2} \rightarrow\|\boldsymbol{\omega}+\epsilon\|^{-2}$. As mentioned in the introduction, due to the inherent noise, this introduces "cloudy" artefacts in the reconstructed phase images.

In the sequel, we shall develop a variational framework to obtain a regularised solution of TIE. 


\section{PHASE RECONSTRUCTION}

Let us now consider the following forward model:

$$
\mathbf{y}=\mathbf{H} \Phi+\mathbf{e},
$$

where $\Phi \in \mathbb{R}^{N}$ is the original phase ${ }^{1}, \mathbf{H} \in \mathbb{R}^{N \times N}$ is the matrix representation of the Laplacian operator with $N$ being the total number of pixels on the detector. In (6), $y$ is the left hand side of (5) that is computed in accordance with (4), and e represents the noise.

In bioimaging applications, it is desirable that the specimens be well-isolated from the background. This necessitates that the reconstruction algorithm preserves the discontinuities across the boundaries. Thus, for regularising the solution, we choose to use the total variation (TV) functional that is defined as

$$
R_{\mathrm{TV}}(\Phi)=\sum_{i \in \mathcal{P}}\left\|\mathbf{L}_{i} \Phi\right\|_{2},
$$

where $\mathcal{P}$ is the set of all pixels and $\mathbf{L}_{i} \Phi \in \mathbb{R}^{2}$ represents the first-order finite differences of $\Phi$ at pixel $i$ along the vertical and horizontal directions. Then, we propose to reconstruct the phase by solving the optimisation problem:

$$
\min _{\Phi} \frac{1}{2}\|\mathbf{H} \Phi-\mathbf{y}\|_{2}^{2}+\tau \sum_{i \in \mathcal{P}}\left\|\mathbf{L}_{i} \Phi\right\|_{2}
$$

To solve (8), we first express an equivalent constraint problem:

$$
\min _{\Phi, \mathbf{u}} \frac{1}{2}\|\mathbf{H} \Phi-\mathbf{y}\|_{2}^{2}+\tau \sum_{i \in \mathcal{P}}\left\|\mathbf{u}_{i}\right\|_{2}: \mathbf{u}=\mathbf{L} \Phi,
$$

where $\mathbf{u}$ is an auxiliary variable and $\tau>0$ is the regularisation parameter. For (9), we introduce the associated augmented Lagrangian (AL) that is of the form

$$
\begin{aligned}
\mathcal{L}_{\mathcal{A}}(\Phi, \mathbf{u}, \boldsymbol{\alpha}) & =\frac{1}{2}\|\mathbf{H} \Phi-\mathbf{y}\|_{2}^{2}+\tau \sum_{i \in \mathcal{P}}\left\|\mathbf{u}_{i}\right\|_{2} \\
& -\boldsymbol{\alpha}^{\mathrm{T}}(\mathbf{u}-\mathbf{L} \Phi)+\frac{\beta}{2}\|\mathbf{u}-\mathbf{L} \Phi\|_{2}^{2},
\end{aligned}
$$

where $\alpha$ is the Lagrange multiplier and $\beta>0$ is the penalty parameter. We then use alternating direction method of multipliers (ADMM) [9] where the main idea is to minimise the $\mathrm{AL}$ functional with respect to each variable as the others are kept fixed. Applying ADMM to AL functional results in the given iterative framework:

$$
\begin{aligned}
& \mathbf{u}^{t+1} \leftarrow \arg \min _{\mathbf{u}} \mathcal{L}_{\mathcal{A}}\left(\Phi^{t}, \mathbf{u}, \boldsymbol{\alpha}^{t}\right), \\
& \Phi^{t+1} \leftarrow \arg \min _{\mathbf{x}} \mathcal{L}_{\mathcal{A}}\left(\Phi, \mathbf{u}^{t+1}, \boldsymbol{\alpha}^{t}\right), \\
& \boldsymbol{\alpha}^{t+1} \leftarrow \boldsymbol{\alpha}^{t}-\beta\left(\mathbf{u}^{t+1}-\mathbf{L} \Phi^{t+1}\right) .
\end{aligned}
$$

Let us now focus on the sub-minimisation problems. We note that the minimisation over $\mathbf{u}$ is separable and is computed by

\footnotetext{
${ }^{1}$ Note that the phase image is thought to be lexicographically reordered.
}

the following vectorial shrinkage:

$$
\mathbf{u}_{i}^{t+1}=\max \left\{\left\|\mathbf{z}_{i}\right\|_{2}-\frac{\lambda}{\beta}, 0\right\} \frac{\mathbf{z}_{i}}{\left\|\mathbf{z}_{i}\right\|_{2}},
$$

where $\mathbf{z}_{i}=\mathbf{L}_{i} \mathbf{x}^{t}+\frac{\boldsymbol{\alpha}_{i}^{t}}{\beta}$. As for the second sub-problem, we remark that $\mathbf{H}$ is a self-adjoint operator (i.e. $\mathbf{H}^{\mathrm{T}}=\mathbf{H}$ ). The minimizer $\Phi^{t+1}$ is computed by

$$
\Phi^{t+1}=\left(\mathbf{H}^{2}+\beta \mathbf{L}^{\mathrm{T}} \mathbf{L}\right)^{-1}\left(\mathbf{H} \mathbf{y}+\beta \mathbf{L}^{\mathrm{T}}\left(\mathbf{u}^{t+1}-\frac{\boldsymbol{\alpha}^{t}}{\beta}\right)\right) .
$$

Assuming periodic boundary conditions, this is directly solved by using the fast Fourier transform [10]. The final step of the ADMM is a trivial update.

\section{EXPERIMENTAL RESULTS}

To test the capability of the technique, we chose to directly operate with real data (sound simulations of image formation in DIC microscopy is rather involved). Paraformaldehyde-fixed unstained HeLa cells - a cell line commonly used in biologywere imaged at room temperature $\left(\sim 22^{\circ} \mathrm{C}\right)$. Images were acquired on a Zeiss Axio Observer Z1 (Carl Zeiss AG, Jena, Germany) equipped with an APlan $10 \times / 0.25$ NA with a DIC analyser II. The defocused images were recorded with a distance of $2 \mu \mathrm{m}$ from the best focus position. We observed that such defocus provides a good compromise for approximation quality and the measurement noise.

For the phase reconstruction, we compared the direct FFTbased method and the proposed approach. We also included the Tikhnonov regularisation with the same $\mathbf{L}$ operator (solved directly as well) in the comparisons for completeness. We manually chose the regularisation parameter (for Tikhonov and our method) such that the best results (in term of contrast and physiological relevance) are obtained. We set $\beta=\tau / 100$ and used 30 ADMM iterations which was observed to be enough for convergence. A JAVA implementation-on a 2.66 GHz Quad-Core Intel Xeon Mac Pro computer-took roughly 3.5 seconds to reconstruct the phase from $891 \times 704$ pixel images.

By looking at the results given in Figure 2, we first observe that the phase images obtained via TIE render some intracellular structures in a more revealing way than DIC images. For instance, the cell nucleolus (indicating concentrated DNA) is more visible in TIE, especially so with our reconstruction method (see the region inside the circles in Figure 2.(e)). Also, our method produces an image where the nucleus is better resolved compared to the other reconstructions (indicated by arrows in Figure 2.(e)). Finally, we note that the background is reconstructed with improved homogeneity and the halos around the cell membrane (see the circles in Figure 2.(f)) are significantly reduced. The latter can be of importance if further morphological analysis of such cells is considered for which a proper segmentation is needed. 

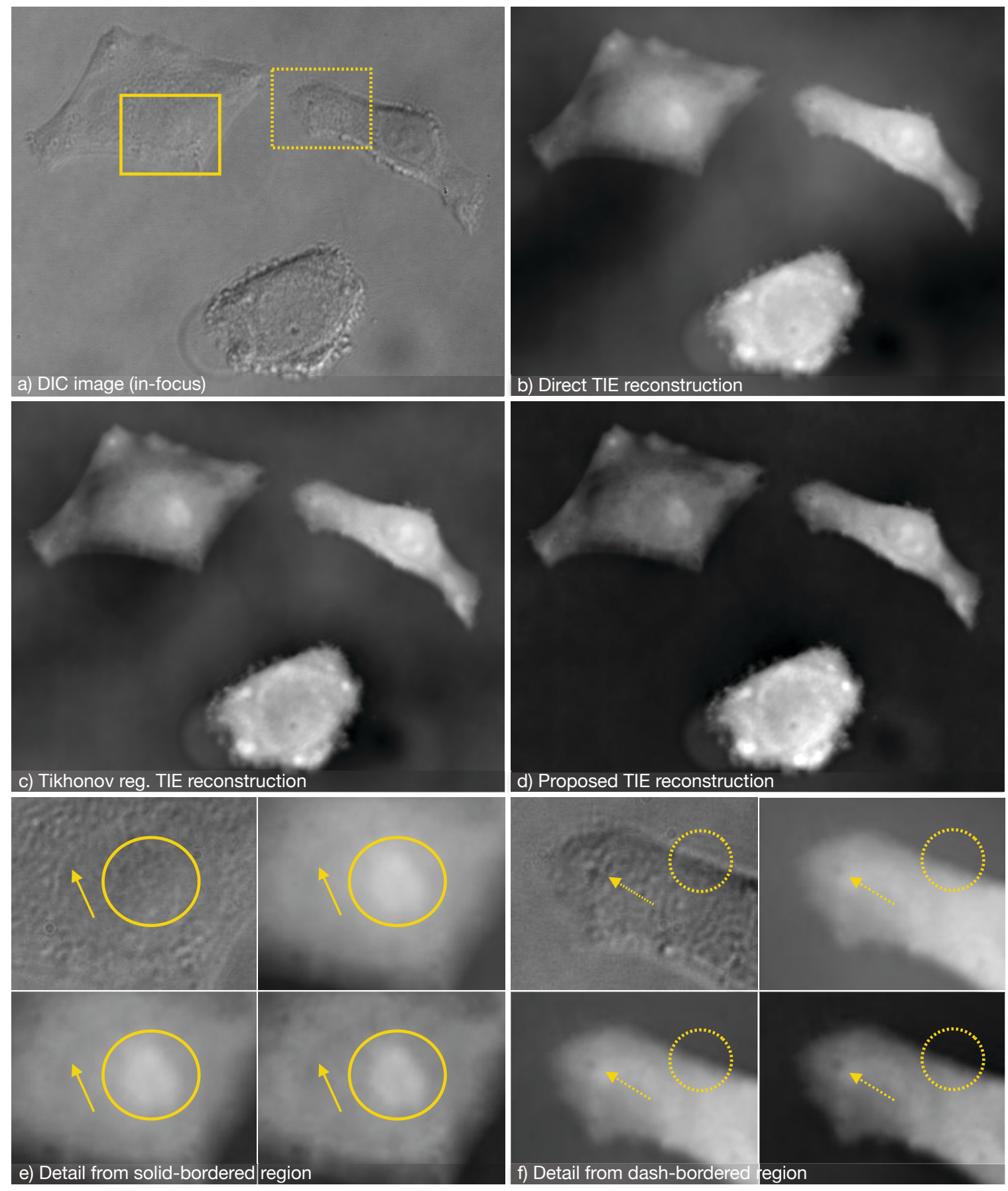

Fig. 2. Phase reconstruction using TIE and DIC: See text for a detailed explanation of the experiment.

\section{CONCLUSION}

We have introduced a variational framework for phase reconstruction using TIE and the DIC microscopy. Our model uses TV regularisation and is solved iteratively via ADMM. Finally, we have demonstrated our phase reconstruction method with experimentally-recorded DIC images.

\section{REFERENCES}

[1] G. Popescu, Quantitative Phase Imaging of Cells and Tissues, Mc, 2011.

[2] M. R. Teague, "Deterministic phase retrieval: a Green's function solution," Journal of the Optical Society of America, vol. 73, no. 11, pp. 1434-1441, 1983. 
[3] M. Beleggia, M. A. Schofield, V. V. Volkov, and Y. Zhu, "On the transport of intensity technique for phase retrieval," Ultramicroscopy, vol. 102, no. 1, pp. 37-49, 2004.

[4] A. Barty, K. A. Nugent, D. Paganin, and A. Roberts, "Quantitative optical phase microscopy," Optics Letters, vol. 23, no. 11, pp. 817-819, 1998.

[5] L. J. Allen and M. P. Oxley, "Phase retrieval from series of images obtained by defocus variation," Optics Communications, vol. 199, no. 1, pp. 65-75, 2001.

[6] D. Paganin and K. A. Nugent, "Noninterferometric phase imaging with partially coherent light," Physical Review Letters, vol. 80, no. 12, pp. 2586-2589, 1998.

[7] S. S. Kou, L. Waller, G. Barbastathis, and C. J. R. Sheppard, "Transport-of-intensity approach to differential interference contrast (TI-DIC) microscopy for quantitative phase imaging," Optics Letters, vol. 35, no. 3, pp. 447-449, 2010.

[8] P. Ferraro, A. Wax, and Z. Zalevsky, Coherent Light Microscopy_Imaging and Quantitative Phase Analysis, Springer, 2011.

[9] S. Boyd, N. Parikh, E. Chu, B. Peleato, and J. Eckstein, "Distributed optimization and statistical learning via the alternating direction method of multipliers," Foundations and Trends in Machine Learning, vol. 3, no. 1, pp. 1-122, 2011.

[10] E. Bostan, U. S. Kamilov, M. Nilchian, and M. Unser, "Sparse stochastic processes and discretization of linear inverse problems," IEEE Transactions on Image Processing, vol. 22, no. 7, pp. 2699-2710, 2013. 